\title{
Ein Beitrag zur Therapie der Ureterenverletzungen bei Laparotomien.
}

\author{
Von \\ Dr. Weinreb, \\ Assistenzarzt der Prof. Landan'schen Erauenklinik in Berino.
}

Eine der unangenehmsten und gefürchtetsten Complicationen bei Ausführung von gynäkologischen Operationen ist die Verletzung der Harnleiter. Während Harnleiterverletzungen bei vaginalen Eingriffen, besonders bei der Totalexstirpation, nicht gerade selten vorkommen, werden sie bei Laparotomien nur in verhältnissmässig geringer Zahl bemerkt, wenigstens soweit das aus der Literatur ersichtlich ist. Im Folgenden sollen nur diejenigen Fälle von Ureterverletzungen bei ventralen Operationen Gegenstand der Betrachtung sein, die als solche schon während des Eingriffs erkannt sind.

Trotz der bedeutenden Fortschritte unserer Technik und trotz aller beim Operiren angewandten Vorsicht scheinen derartige unangenehme Ereignisse nicht gänzlich vermeidbar zu sein. Die Zusammenstellung der Ureterenverletzungen ergiebt, dass diese Verletzung bei der Exstirpation mannigfacher Tumoren sich ereignen kann. Hauptsächlich kommen in Betracht: intraligamentäre Tumoren, maligne Neoplasmen, Ovarialtumoren, uniter diesen besonders das Kystoma proliferum papillare (Tauffer, Dieses Archiv Bd.XLVI. S. 537), ektopische Schwangerschaften, Myome, Geschwülste des Beckenzellgewebes und Pyosalpingen mit parametritischen Narben in der Umgebung des Ureters (J. Veit, Zeitschrift für Geburtsh. und Gynäk. Bd. XXXI. S. 456).

Die anatomischen Veränderungen, die durch die erwähnten Zustände herbeigeführt werden, bestehen in Verlagerung und Fixirung des Harnleiters, und letztere Anomalien sind allermeist die disponirenden Momente zur Verletzung desselben. Trotzdem wohl ein Jeder mit solchen Abnormitäten rechnet und bei den genannten 
Zuständen die nöthige Vorsicht verdoppelt, geschieht es dennoch, dass die Verletzung des Ureters manchmal unvermeidlich wird. Man begegnet nämlich bei Operationen von Bauchgeschwülsten mitunter Fällen, wo es nothwendig ist, die überall adhärente Geschwulst mit mehr oder weniger Gewalt aus der Tiefe des Beckens $\mathrm{zu}$ entwickeln. Bei solcher Gelegenheit kann leicht ein grösseres Gefäss durchreissen und eine dadurch entstandene Blutung das ganze Gesichtsfeld in der Tiefe überschwemmen. In diesem kritischen Moment, in welchem man durch rasches Comprimiren oder Ligiren der Blutung Herr werden muss, kann der Ureter verletzt werden, besonders dann, wenn er irgendwie dislocirt ist und an der blutenden Stelle seineAnwesenheit nicht vermuthet werden konnte.

Ausser diesen unbeabsichtigten Verletzungen giebt es auch Fälle, in welchen der Ureter mit klarer Erkenntniss der Situation durchschnitten wird, z. B. wenn er von carcinomatösen Wucherungen umstrickt ist.

Der Ureter kann in dreifacher Weise verletzt werden, er kann

1. einfach unterbunden,

2. theilweise angeschnitten,

3. vollständig durchschnitten, bez. auf einer kleineren oder grösseren Strecke resecirt werden, letzteres besonders bei Entfernung übermässig grosser Geschwülste, auf deren Höhe der Ureter bogenförmig verläuft.

Um Verletzungen des Ureters zu vermeiden, ist es nöthig, eine ausgedehnte Ablösung der Blase und Ureteren vom Uterus und den Ligamenta lata bei allen Totalexstirpationen vorzunehmen, sowie möglichst isolirte Unterbindung einzelner Gefässe der Verwendung von Massenligaturen vorzuziehen und sonst bei allen Ausschälungen sich dicht an das Object und an den Uterus zu halten. Kelly schlug vor, in die Harnleiter Bougies einzuführen, um ihre Lage während einer Hysterectomie oder Entwickelung von adhärenten Geschwülsten zu markiren. Tauffer räth, immer die Möglichkeit einer Unterbindung des Ureters vor Augen zu behalten, besonders wenn man Unterbindungen tief im Becken ausführen musste. Er empfiehlt daher eine scharfe Controlle, d.h. man soll in solchen Fällen jedesmal die Palpation der Ureteren vornehmen, da der Ureter, wenn er irgendwo ligirt ist, in seinem Lumen noch während der Operation eine Stauung aufweist; wenn man demnach die Abtastung in einer entsprechenden Höhe beginnt, so erkennt man an der einen oder der anderen Seite der Wirbelsäule in der 
Form eines starken cylindrischen Stranges die Ligirung des Ureters. Diesem Umstande, sagt Tauffer, dass er nach Beendigung der Operation den Verlauf der Ureteren in Augenschein nimmt, verdankt er es, dass er wiederholt noch rechtzeitig, wenn auch mit Schwierigkeit, die Unterbindungsstelle löste.

Wohl nicht immer ist man in der Lage, die nicht mit $\triangle$ bsicht herbeigeführte Verletzung des Ureters sofort zu erkennen und dann alsbald zur Beseitigung des Schadens zu schreiten. Missliche Wundverhältnisse bilden für diese Erkenntniss sicher nur zu häufig ein unüberwindliches Hinderniss. Ist aber die Verletzung des Ureters erkannt, so hängt es von dem Orte der Verletzung ab, wie man sich dabei verhalten soll. -

Blumenfeld (Ureterenverletzungen bei Laparotomien, Münchener med. Wochenschrift Bd. XLV. S. 992) hat das Verdienst, aus der Literatur die verschiedenen Mittheilungen über Ureterenverletzungen bei Laparotomien zusammengestellt zu haben. Im Folgenden sollen dic nach dem Aufsatze Blumenfeld's veröffentlichten Ureterenverletzungen bei ventralen Operationen und das dabei eingeschlagene Verfahren in Kürze angeführt, sodann ein Fall geschildert werden, der in unserer Klinik zur Beobachtung gekommen ist.

Fall von Ligaturlösung nach Ureterunterbindung:

Purcell (Brit. Gyn. Journ., Vol. LIV, pap. 174) theilt einen Fall mit, in welchem bei einer Totalexstirpation des. Uterus wegen verjauchten Myoms beide Ureteren in Ligaturen gefasst worden waren. Als nach 58 Stunden bei völliger Anurie die ersten Symptome von Urämie eintraten, wurde das Abdomen neuerdings geöfinet und unter Verfolgung der nach oben stark erweiterten Ureteren die Ligaturen, welche die Uterinarterien gefasst hatten, gelöst, ohne dass eine Blutung aus den Gefässen eintrat. Die Blase füllte sich sufort mit Harn, da beide Ureteren nicht verletzt waren, und es trat vollkommene Heilung ein.

Fälle von Ureterverletzungen, bei denen der Ureter in die Blase implantirt wurde:

Funke (Zeitschrift für Geburtshilfe und Gyn., Bd. XXXIX, S. 485) berichtet aus der Freund'schen Klinik über zwei Fälle von Ureterenverletzungen bei abdominaler Totalexstirpation carcinomatöser Uteri. In dem einen Falle wurde der Ureter, der mitten durch Carcinommassen ging, bewusst durchschnitten mit 
sofortiger nachfolgender Implantation in die Blase: Heilung. Beim zweiten Falle wurde wegen völliger 48 stündiger Anurie die secundäre Laparotomie ausgeführt, und da man nicht erkennen konnte, ob ein oder beide Ureteren versehlossen waren, so wurden beide resecirt und in die Blase eingepflanzt. Trotzdem bildete sich späterbin eine Ureterfistel aus. Eine weitere Beobachtung der Kranken war unmöglich, da die Patientin nach ihrer Entlassung bald an Eiterfieber starb.

Gräupner (Inaugural-Dissert,, Strassburg 1897) berichtet über einen von Bayer (Strassburg) operirten Fall, in welchem bei abdominaler Myomektomie der Ureter dicht an der Blase durchtrennt wurde. Derselbe wurde sofort wieder in die Blase eingepflanzt und die Einpflanzungsstelle dureb Ligament- und Peritonealnähte überdeckt und die in den Ureter gelegten Fäden zur Harnröhre herausgeleitet. Völlige Heilung unter Gebrauch des Verweilkatheters.

Lotheisen (Wiener Klin. Wochenschrift, Bd. XII, S. 883) berichtet einen Fall von absichtlicher Resection eines $3 \mathrm{~cm}$ langen Ureterstückes bei abdominaler Totalexstirpation des carcinomatösen Uterus; die betreffende Stelle war vollständig von Carcinommassen umgeben, so dass die Resection nothwendig war. Es wurde sofort das centrale Ende des Ureters in die Blase implantirt, die Peritonealhöhle geschlossen und die Wundhöhle nach der Scheide zu drai-. nirt. Heilung.

Wertheim (Monatsschrift für Geburtshilfe und Gyn., Bd. X, S. 523) bespricht zunächst zwei Fälle von beabsichtigter Ureterverletzung. In dem einen Falle von Excision eines Blasenearcinoms, das um die Mündung des Ureters sass, implantirte er nach $\mathrm{Ab}-$ tragung eines $21 / 2 \mathrm{~cm}$ langen Ureterstückes den Ureter auf rein extraperitonealem Wege in die Blase. Nach $2 \frac{1}{2}$ Monaten starb die Operirte, und es fanden sich bei der Section im Operationsgebiet völlig normale Verhältnisse. Im zweiten Falle, in welchem ein Ovarialsarcom den Ureter umwachsen hatte, wurde ein grösseres Stück vom Colon descendens und zugleich ein $6-7 \mathrm{~cm}$ langes Ureterstück excidirt; das distale Ende wurde in die Blase eingenäht, vaginale Drainage, in der Folge Ureterscheidenfistel. - Ein dritter Fall von Implantation in die Harnblase wurde in einem Falle von Myomoperation ausgeführt, bei welcher der Ureter unabsichtlich verletzt worden war. Gleichfalls Heilung. In allen 
drei Fällen ragte ein Stumpf des Ureters in die Blase vor, an dem sich Harnentleerung und Peristaltik beobachten liessen.

Baldwin (Phil. Med. Journ. 1899, referirt Monatsbericht über die Krankheiten des Harn- und Sexual-Apparates, Vol. IV, pag. 307) excidirte gelegentlich einer Hysterectomie wegen Fundussarcoms ein $1 \frac{1}{2}$. Zoll langes Stück des rechten Ureters. Behufs Implantation des beträchtlich verkürzten. Ureters in die Blase wurde die Blasenwand durch eine eingeführte Zange vorgeschoben und gegen diese Zange incidirt. Hierauf wurde das Ureterende in die Blasenöffnung hineingezogen, und die Blasenwand mit dem Ureter durch eine Katgutnahtreihe vereinigt, Heilung.

Polk (Amer. Gyn. and Obst. Journ., Vol. XII, pag. 200) resecirte bei einer abdominalen Hysterectomie wegen Carcinom ein zolllanges Stück des Ureters und nähte das Ureterende in die Blase ein. Genesung.

Die Vereinigung der durchtrennten Uretertheile wurde in folgenden Fällen ausgeführt:

Paroschin (Wratsch 1897, No. 19 ref: Centralblatt für Chirurgie, Bd. XXV, S. 819) berichtet über einen Fall von Durehschneidung: des Harnleiters bei Exstirpation einer intraligamentären Cyste (Klinik SIawjansky). Die Verletzung wurde sofort am tropfenweise abfliessendon Urin bemerkt. Durch vier dünne Seidennähte, die nur Serosa und Muscularis fassten, wurden die Enden vereinigt. Bis zum 5. Tage häufiger Harndrang, Urinmenge 100-300 ccm, geringe Beimengung von rothen Blutkörperchen. Am 12. Tage Urin normal, nach fünf Wochen wird Patientin geheilt entlassen.

Winslow (Ann. of Surg. 1898, Januar, ref. Centralblatt für Gyn., Bd. XXII, S. 1074) berichtet über zwei Fälle von Verletzungen dès Ureters bei Totalexstirpation des Uterus wegen Fibromyomen. Bei dem einen Falle lag vollständige Durehtrennung, beim anderen nur seitliche Verletzung vor. Beide Male wurde die Naht ausgeführt und Heilung erzielt, doch bildete sich im letzteren Falle eine Infiltration im breiten Mutterbande an der Nahtstelle, bei deren Incision von der Scheide aus sich urinös riechende Flüssigkeit entleerte. Die Vereinigung erfolgte secundär. Das distale Ende wurde in das proximale invaginirt und festgenäht, wobei das letztere durch einen seitlichen Schlitz erweitert wurde. Noble (Med. News, New-York, Vol LXXIII, pag. 854) ver- 
letzte den Ureter bei der Exstirpation eines intraligamentären Myoms. Das vesicale Ende wurde geschlitzt und das renale in diesen Schlitz mittelst einer Matratzennaht eingenäht und mit feinen Seidennähten aussen geschlossen. Die Nahtstelle wurde mit Peritoneum bedeckt. Die Vereinigung geschah über einem Ureterkatheter; Heilung. In einem zweiten Falle wurde bei Exstirpation eines Ovarialsarcoms, das sich subperitoneal entwickelt hatte, der Ureter in einer Ausdehnung von $1 \frac{1}{2}$ Zoll resecirt. Die Vereinigung geschah in gleicher Weise wie beim ersten Falle, nur musste zur Vermeidung einer Dehnung der Ureterenden die Blase von der Scheide und vom horizontalen Schambeinaste abgelöst werden. Auch hier erfolgte Heilung.

Lavisé (Annal. des mal. des org. gén.-ur. Tom. XVII, pag. 724) durchschnitt den rechten Ureter in einem Falle von abdominaler Hysterectomie wegen Uteruscarcinoms. Da die Verletzung zu weit von der Blase entfernt war, musste auf eine Implantation des proximalen Endes in die Blase verzichtet werden, und es wurde über einen in beide Ureterenden eingeführten Harn. leiterkatheter die Vereinigung der Ureterenden durch die Naht bewerkstelligt, über der letzteren das Peritoneum genäht, hierauf die Stelle der Ureternaht nach der Scheide drainirt. Es stellte sich Harnabfluss ein, aber die Fistel schloss sich nach 10 Tagen von selbst. Der Ureterkatheter wurde nach 2 Tagen entfernt.

Mac Managle (Amer. Journ. of Med. Sciences, Vol CXVIII, pag. 684) berichtet über drei Fälie von Ureterenverletzung bei abdominaler Exstirpation von Uterusmyomen. In allen drei Fällen wurde das proximale Ureterende in das distale invaginirt und durch Nähte befestigt. Eine der. Operirten starb alsbald nach der Operation an Shok, in den beiden anderen Fällen war der Verlauf ein günstiger, und es trat völlige Heilung ein.

Markoe und Wood (Uretero-ureteral anastomosis, Annal. of Surgery 1899, Heft 6) excidirten bei Exstirpation eines intraligamentären Myoms ein $1 \frac{1}{2}$ Zoll langes Stück des rechten Ureters. Es kam zur völligen Anurie - durch den Verschluss des geknickten linken Ureters. Das Abdomen wurde wieder geöffnet und zunächst der linke Ureter aus seiner Knickung befreit. Rechterseits wurde über einem Katheter das proximale Ende des Ureters in das distale invaginirt und dann durch Circulärnaht befestigt. Drainage, Heilung.

Schauta (Centralblatt für Gynäk. XXIII. S. 425) berichtet 
über eine Ureterenverletzung bei Exstirpation eines pseudo-intraligamentären extrauterinen Fruchtsackes, die er bei Revision des Operationsfeldes fand. Ein Stück des Ureters stand mit der Blase in Verbindung, dann fand sich ein Zwischenraum von mehreren Centimetern, doch fehlte Nichts vom Uretergewebe. Er brachte die beiden Ureterenden so aneinander, dass er an dem oberen Ende eine Fadenschlinge durchzog und beide Enden des Fadens etwa 1 bis $11 / 2 \mathrm{~cm}$ unterhalb der Schnittfläche des unteren Ureterendes so in das untere Ende einführte und durch die Wand durchzog, dass sich das obere Ureterende in das untere einstülpte. Die Fadensehlinge wurde dann geknüpft (Methode von Kelly). Die Nahtstelle wurde mit Peritoneum überdeckt und subperitoneal nach der Bauchwunde zu drainirt. In den ersten Tagen trat Oligurie auf, die vom dritten Tage an normaler Harnmenge Platz machte. Heilung.

Die Nephrektomie wurde primär oder secundär von folgenden Autoren gemacht:

Rumpf (Dieses Archiv. Bd. LV. S. 182) veröffentlicht einen Fall von Ureterverletzung, die die Nephrectomie nöthig machte. Die Operation betraf eine Multipara im VII.-VIII. Schwangerschaftsmonat mit Portiocarcinom, das in das rechte Ligam. sacrouterinum übergriff. Wegen der Möglichkeit der Lebensfähigkeit der Frucht wurde mit der Sectio caesarea begonnen. Bei der folgenden Totalexstirpation des Uterus wurde bei der Durchtrennung des verkürzten rechten hinteren Bandes der rechte Ureter unterbunden und durchschnitten. Wegen der langen Dauer der Operation an einer vorher durch Blutungen geschwächten Patientin schien Rumpf der Versuch, das centrale Ende frei zu machen und in die Blase einzunähen, zu gewagt; es wurde daher die Bauchhöhle sofort geschlossen und die rechte Niere von hinten exstirpirt. Heilung.

Krüger (Centralblatt für Chirurgie. Bd. XXV. S. 1054) beschreibt einen Fall von Laparotomie bei intraligamentär entwickelter Ovarialcyste, in welchem von dem mit der Sackwand innig verwachsenen Ureter trotz aller Vorsicht bei der Loslösung ein circa $8 \mathrm{~cm}$ langes Stück abriss. Wegen der Grösse des fehlenden Stückes war eine Vereinigung der Ureterenden unmöglich; die Einpflanzung: des centralen Stückes in den Darm schien bedenklich wegen der Gefahr einer aufsteigenden Pyelitis; bei Anlegung einer Ureter- 
bauchfistel hätte später auch nur durch Nephrectomie Heilung erzielt werden können; es wurde daher die Nephrectomie sofort vorgenommen. Heilung obne Zwischenfall. - In einem zweiten Falle war der Ureter bei einer Laparotomie wegen doppelseitiger allseitig adhärenter Adnextumoren verletzt worden. Nach 8 Tagen zeigten sich die in die Bauchhöhle gelegten Tampons urinös durchtränkt. Nach 4 Wochen war die Wunde geschlossen, bis auf eine kleine Fistel, aus der sich Urin entleerte; per vias naturales gingen $400-700 \mathrm{ccm}$ Harn ab. Nach anfänglichem Wohlbefinden traten später urämische Erscheinungen auf, die zur Nierenexstirpation drängten. Bei der Nephrectomie fand sich die linke Niere - die Seite der Verletzung war mittelst Kathetcrismus der Ureteren sichergestellt worden - auf das Doppelte vergrössert und mit linsen- bis bohnengrossen Eitercysten besetzt, sowie Nierenbecken, Nierenkelche und Anfangstheil des Ureters stark erweitert.

Ein weiterer Fall von secundärer Nephrektomie stammt von Noble. Noble (Pennsylvania Med. Journ. No. 8) excidirte bei Exstirpation eines extra-uterinen Fruchtsackes und eines intra-ligamentären Ovarialtumors den grössten Theil des Ureters und nähte denselben, da eine Implantation in die Blase wegen der Kürze des restirenden Ureterstückes nicht möglich war, in die Bauchwunde ein. Später wurde die Niere exstirpirt; Heilung. - Noble zieht die Nephrektomie der Einpflanzung in den Darm vor.

In folgenden Fällen wurde die einfache Unterbindung des renalen Abschnittes des durchschnittenen Ureters vorgenommen:

Bastianelli (Risconto di Laparotomia, Ann. di Obst. e di Gin. 1896, No, 2) unterband in einem Falle nach einer bei einer Laparotomie erfolgten Ureterverletzung das centrale Ende und versenkte es; dadurch kam es zu einer aseptischen Vérödung der Niere, die ohne Beschwerden vor sich ging.

Füth (Centralblatt für Gyn., Bd. XXII, S. 729) beschreibt einen Fall, in welchem er bei Exstirpation eines Tumors ein 10-12 cm langes Stück des Úreters mitentfernte. Es handelte sieh um eine $491 / 2$ Jahre alte Nullipara, die seit 19 Jahren eine allmählich wachsende Geschwulst im Leibe bemerkte. Der Leib war colossal durch ein Fibroid aufgetrieben; ausser Menorrhagien 
stellten sich Athembeschwerden, Urinverhaltung und sehr starke Schmerzen ein. Bei der Herauswälzung des Myoms entwickelte sich von hinten her und rechts eine zweimannskopfgrosse Ovarialcyste, welche mit zwei grossen Klemmen abgeklemmt und abgeschnitten wurde. Hieraul liess sich das Myom, das an seiner Basis subperitoneal sich derart ausgebreitet hatte, dass das ganze Beckenperitoneum mit in die Höhe genommen war, leicht entwickeln. Bei den Lnterbindungen wurde ein von der Wirbelsäule her zur Geschwulst hinziehendes Gefässbündel mit fingerdicken Venen nach genauer Untersuchung, ob sich kein Darm darin befand, doppelt unterbunden und durchschnitten. Wie sich später herausstellte, enthielt dieses Gebilde die Vasa spermatica interna dextra und den rechten Ureter. Nach Amputation der Geschwulst bemerkte man ein über derselben bogenförmig verlaufendes Gebilde, das sich bei genauer Besichtigung an seiner Form, Dicke und Schleimbautauskleidung als Ureter erkennen liess. Das centrale Ureterende war in dem erwähnten Gefässbündel fest zugeschnürt, das peripherisehe Ende $\mathrm{kam}$ nicht $\mathrm{zu}$ Gesicht. Nach reiflicher Ueberlegung wurde das centrale Ende, da dasselbe wegen seiner Kürze in die Blase nicht eingepflanzt werden konnte und die Implantation in den Darm die Gefahr der aufsteigenden Infection mit sich bringt, nochmals fest unterbunden und die Bauchhöhle geschlossen. Die Heilung erfolgte, allerdings unter leichten Fieberbewegungen ohne weitere Störung. Allmählich bildete sich ein kindskopfgrosser Hydronephrosensack, der aber völlig symptomlos und der Operirten sogar unbekannt blieb und daher nicht exstirpirt wurde. - Füth wollte die Niere nicht unmittelbar völlig opfern, sondern nur ausschalten in der Absicht, wenn sich die andere Niere als ungenügend functionirend erweisen sollte, sie durch Herstellung einer Nierenbeckenfistel wieder functionsfähig machen.

Phenomenow: (Monatsschrift für Geburtshilfe und Gyn., Bd. XII, S. 233) berichtete in der geburtshilflich-gynäkologischen Gesellschaft zu St. Petersburg über einen Fall von Ureterenverletzung bei abdominaler Entfernung von Geschwülsten beider Ovarien. Die Operation betraf eine 35 jährige Nullipara, die über Schmerzen im Unterleib, über Empfindung ron Schwere und von Druck nach abwärts und über erschwertes Uriniren klagte. Die Untersuchung per vaginam ergab im rechten und linken Scheidengewölbe fluctuirende Tumoren, die dem Uterus dicht anlagen, unheweglich und tief in den Ligamentis latis sassen. Die Entwickelung der Tumoren 
war wegen ihrer ausgedehnten Verwachsungen mit Netz und Darm äusserst schwierig. Da der Operateur das Vorhandensein von eitrigem Inhalt in den Geschwülsten vermuthete, suchte er dieselben in toto auszuschälen. Das gelang ihm auf der linken Seite; auf der rechten Seite jedoch, wo der Tumor viel tiefer sass, bemerkte er während der Lösung der Verlöthungen plötzlich zwischen der Geschwulst und ihrer Kapsel angehäufte helle Flüssigkeit: es erwies sich, dass der rechte Harnleiter durchschnitten war. Da ein Theil desselbẹn herausgeschnitten war, konnten die durchschnittenen Enden des Harnleiters nicht zusammengebracht und wegen des grossen Abstandes das centrale Ende auch nicht in die Blase eingenäht werden. Eine Implantation in den Darm oder in die Bauchwunde wollte Operateur nicht vornehmen, da solche Fälle mit der Zeit die Exstirpation der entsprechenden Nieren zu erheischen pflegen oder sogar tödtlich enden können. Darum begnügte er sich, den centralen Theil des Ureters so hoch wie möglich abzubinden. Die Heilung verlief ohne Zwischenfall, wahrscheinlich entwickelte sich Atrophie in der rechten Niere.

Im Anschluss an die letzten drei Fälle will ich über einen Fall aus unserer Klinik berichten:

Frau B. P., J.-No. 6850, Arbeitersfrau, 39 Jahre alt, 3 Partus, kein Abort. Menstruation - erste mit 13 Jahren - stets schmerzlos, alle 4 Wochen, von 6-8tägiger Dauer. Patientin war nie bei einem Arzte, fühlte sich immer vollständig gesund. Die letzten 6 Menstruationsblutungen ziemlich profus, in den letzten Wochen unregelmässige Blntungen sowie Blutungen post coitum, keine Schmerzen.

Status: Grosse, gut genährte, kxäftige Frau. Vagina weit, im Scheidengewölbe fühlt man Nichts von der Portio, dagegen ein kraterförmiges, im Niveau der umgebenden Scheide gelegenes blutendes Geschwür, ans dem sich bröcklige Massen leicht loslösen. Der Uterus ist vergrössert, hart, ohne Buckel, links und hinter ihm anscheinend keine Resistenzen, rechtes Parametrium infiltrirt.

Diagnose: Carcinoma cervicis.

17. 5. 1900. Operation (Prof: Landau). Da alle Versuche, die Cervix mit Muzeuxs zu fassen, misslingen, indem das Gewebe ohne Weiteres abbricht, wird von der Operation auf vaginalem Wege Abstand genommen und mit Rücksicht auf den guten Allgemeinzustand zur abdominalen Totalexstirpation übergegangen. Schnitt in der Linea alba. Nach Eröffnung des Peritoneums wird der Uteruskörper mittelst einer Klemmzange hervorgezogen, sodann die Unterbindung der Ligamenta infundibulo-pelvica beiderseits vorgenommen. Nachdem der Uterus hierdurch ziemlich mobil geworden ist, wird oberhalb der Blasengrenze ein horizontaler Schnitt durch das Peritoneum auf der Vorderfläche des Uterus geführt und beiderseits nach den Ligamenta lata zu bogenförmig verlängert. Hierauf wird die hintere Peritonealbekleidung, und zwar etwas tiefer als vorne, durchtrennt. Nunmehr zeigt sich, dass die carcinomatöse 
Infiltration bereits in beide Parametrien vorgedrungen ist. Bei den Versuchen, hier im Gesundèn vorzudringen, reisst plötzlich der ziemlich stark angezogene Uterus von seiner morschen carcinomatösen Basis ab. Nach vorheriger Anlegung von Klemmen von der Vagina aus lateral im makroskopisch gesunden Gewebe sucht nun der Operateur möglichstalles Kranke theils vom Abdomen, theils von der Vagina aus zu entfernen, was sich ausserst schwierig gestaltet, da das Gewebe sehr brüchig ist und die vielen kleinen Knötchen ohne Weiteres abbröckeln. Schliesslich bleibt nur noch rechts ein grösserer Knoten im parametranen Gewebe zurück, der unterbunden und dann abgetragen wird; die Fadenenden werden in die Vagina geleitet. Bei der Revision sieht man rechterseits einen dem Verlaufe des rechten Ureters entsprechenden Strang, der mit dem freien Ende an der Stelle des ursprünglich rechts befindlichen grossen Carcinomknotens endigt. Eine sofort vorgenommene histologische Untersuchung eines exstirpirten Stückchens dieses Stranges zeigt, dass man es in der That mit dem Ureter zu thun hat. Die Einpflanzung desselben in die Blase erweist sich wegen seiner Kürze als unmöglich; ebensowenig ist eine Ureter-Ureteroanastomose ausfürbar, da das vesicale Ende des Harnleiters überhaupt nicht auffindbar ist. In Anbetracht der langen Dauer der sehr eingreifenden Operation entschliesst, sich der Operateur, das centrale Ureterende lediglich fest zu unterbinden, um eventuell später die Nephrectomie auszuführen. Darauf Schluss der Bauchwunde mit tiefgreifenden Silber- und oberflächlichen Silkwormnähten. - Der Verlauf nach der Operation war ein über Erwarten guter. Es stellten sich zwar am 2. und 3. Tage post operationem in der rechten Nierengegend dumpfe Schmerzen ein, sie verschwanden aber spontan; auch traten von Zeit zu Zeit Temperatursteigerungen bis $38^{\circ}$, einmal (am 14. Tage) $39^{\circ}$ und Erbrechen auf, Patientin fühlte sich aber trotzdem wohl. Tumorbildung in der rechten Nierengegend nicht nachweisbar. Der Urin war in den ersten zwei Tagen blutig, die Harnmenge betrug in den ersten Tagen $700-800 \mathrm{ccm}$, sie nahm allmälig zu, so dass sie vom 10 . Tage ab täglich $1000-1200 \mathrm{~g}$ ausmachte. Patientin wird bei bestem Woblbefinden geheilt entlassen. -

Wie aus dem Angeführten ersichtlich, richtet sich die Behandlung der Verletzungen des Ureters in erster Linie nach der Art und dem. Umfange derselben. Ist der Uterus in eine Ligatur gefasst, so braucht diese bloss gelöst zu werden. Ist ein Einriss entstanden, handelt es sich um eine seitliche Verletzung, so wird eine Naht nach Art der Lambert'schen Darmnaht genügen. Anders bei der queren Durchtrennung. Wie man sich in solchen Fällen verhalten soll, hängt vor Allem von dem Ort der Verletzung ab. Geschah die Verletzung in der Nähe der Blase, so kann die Einpflanzung in die Blase oder die Vereinigung der beiden Ureterenden vorgenommen werden. Die meisten Autoren (Veit, Sänger, Büdinger, Lotheissen, Wertheim, Knaggs u. A.) ziehen das Einnähen des Ureters in die Blase vor und halten dieses Verfahren für die zweckmässigste Methode.

Die Implantation des' Ureters in die Blase kann aut rein 
intraperitoneale oder intra-extraperitoneale Weise nach Witzel bewerkstelligt werden. Letztere Methode wird allgemein bevorzugt. Die Nachtheile jeder rein intraperitonealen Implantation beruhen auf der Gefahr einer Darmeinklemmung, zu welcher der strangförmig die Höhle des kleinen Beckens durchziehende Ureter leicht Veranlassung geben kann, und der möglichen Abknickung des Harnleiters selbst bei eintretender Schwangerschaft; ferner besteht bei dieser Methode die Gefahr des Austrittes von Urin in die Bauchhöhle, falls auch nur eine einzige Naht ausreissen sollte, Dem gegenüber bietet die intra-extraperitoneale Methode von Witzel eine Reihe von Vortheilen. Der intraperitoneale Akt dieses, Verfahrens, der sich auf die Aufsuchung des Ureters bezieht, kommt bei frischen Verletzungen während einer Laparotomie selbstverständlich nicht in Betracht, da wir den Ureter bereits vor uns baben, sodass die ganze eigentliche Operation der Uretereinpflanzung vollkommen extraperitoneal sich.vollzieht. Das bis zur Implantation durch eine Ligatur verschlossene obere Ureterende wird mit einer langen Zange, welche extraperitoneal von der Blasengegend her oberhalb der Linea innominata über die Darmbeinhöhlung geführt wird, unter dem Peritoneum nach aussen geleitet und, nachdem das Peritoneum vollkommen abgeschlossen ist, in den dislocirten, ebenfalls extraperitoneal liegenden Theil der Blase folgendermaassen eingepflanzt: nicht der Ureter wird durch starken Zug der Blase genähert, sondern umgekehrt kommt die freigemachte, bis an das Darmbein dislocirte Blase in die Nähe des Ureters, wodurch ohne besondere Spannung oder Zerrung die Implantation des Ureters vor sich gehen kann. Die Blase verträgt nämlich ohne Störung ibrer Function diese Dislocation, wie ja bekanntlich Myome des Uterus und andere Tumoren des kleinen Beckens enorme Verschiebungen und Dehnungen der Blase ohne Functionsalterationen zu Stande bringen. Gegenüber dem schräggeschnittenen Ureterende wird auf einem vorgedrängten. Katheter ein kleines Loch in der Blase angelegt und der schräge Schlitz der Ureterschleimhaut mittelst Ringnaht aus feinstem Catgut mit der Blasenschleimhaut vernäht; alsdann die musculäre Wand des Harnleiters durch eine weitere Catgut-Nahtreihe mit der Blasenwand vereinigt. Die Blasenwand wird dabei parallel zum Ureter in je eine Längsfalte erhoben, und die Höhen der Falten werden so vernäht, dass ein Schrägkanal von $4 \mathrm{~cm}$ Länge entsteht, in welchem der Ureter bis zur Einmündung in die Blase verläuft. Witzel sieht in der 
schrägen Zuschneidung des Ureterendes einen Schutz gegen ringförmige Stenosenbildung und will mit dem Hinaufnähen der Blasenwand eine Nachbildung der normalen Einmündungsweise erzielen. Die Implantationsstelle wird nach aussen drainirt, in die Blase ein Verweilkatheter eingeführt. Die Erfolge dieses Verfahrens sind so vortreffliche, dass man es wohl als die Normalmethode für die Ureterimplantation in die Blase bezeichnen darf.

Erwähnenswerth ist die Technik der Implantation nach Bayer (Inaug.-Diss., Strassburg, Gräupner 1897). In einem Falle, in welchem er bei abdominaler Myomectomie den linken Ureter nahe an der Blase durchschnitt, machte er die. Harnleitereinpflanzung so, dass er eine lange Klemmpincette durch die Urethra in die Blase führte, alsdann den linken Zipfel des Organs vordrängte und auf der Pincette einschnitt. Dann fasste er mit der durch das Loch herausgeführten Klemme den Ureter bez. 3 Seidenfäden, welche vorher an den centralen Ureterstumpf angelegt waren und zog den Ureter in die Blase und die Seidenfäden zur Urethra heraus, wodurch sich der Ureter sehr leicht in die Blase invaginiren liess. Einige feine Knopfnähte fixirten ihn an diese. Die Gewebe incl. Peritoneum wurden darauf in drei Schichten über der Implantationsstelle vernäht, so dass dieselbe vollständig extraperitoneal gelagert wurde. Völlige Heilung unter Gebrauch des Verweilkatheters. -

Die Vereinigung der Ureterenenden ist nicht Concurrenzmethode der Ureterimplantation in die Blase, da letzteres Verfahren die beste und erprobteste Methode ist, um verletzte Ureteren wieder functionsfähig zu machen; sie kommt vielmehr nur dann in Betracht, wenn diese unausführbar ist. Je weiter nämlich nach der Niere hin der Ureter durchschnitten wird, je kürzer also der Stumpf des Ureters ist, um so mehr wird die Implantation in Frage gestellt werden, wenn auch eine ausgedehnte seitliche Dislocation der Blase nach Witzel das Operationsgebiet für die Implantation mehr ausdehnt, wie man a priori annehmen sollte.

Für die Vereinigung durchtrennter Ureterenden ist eine Reihe von Methoden angegeben:

1. Circuläre Naht der a) quer abgeschnittenen Enden (Tauffer, Dieses Archiv Bd. XLVI, Paroschin, s. o., Lavisé, s. o.); b) der abgeschrägten Enden (Bovée, Ann. of surgery. 1897).

2. Invagination a) des distalen Endes in das etwas geschlitzte Lumen des proximalen (Winslow, s. o.); b) des proximalen 
Endes in das distale (Mac Monagle, Markoe und Wood, Sehauta, s. 0.).

3. Seitliche Implantation a) des oberen Endes in das untere, dessen freie Oeffnung abgebunden wird (Emmet, Amer. Journ. of obstetr. 1895, und van Hook, Journ. of the Amer. med. assoc. 1893, Noble,s. o.); b) oder umgekehrt, des peripherischen in das centrale (Kelly, Annal of surgery. 1894. I); c) Monari und Tuffier haben, um Stricturen zu vermeiden, empfohlen, eine Anastomose herzustellen, nach Art der lateralen Enteroanastomose mit Verschluss beider Stumpfenden.

So viele Methoden es aber auch geben mag, die Implantation in die Blase ist der Ureternaht jedenfalls vorzuzieben, da bei der letzteren einerseits die Technik wegen der Dünnheit der Ureterwandungen schwieriger und die Festigkeit der Naht problematischer ist, andererseits eine secundäre Verengerung in der Vereinigungsstelle sich trot\% aller Vorsichtsmaassregeln leicht ausbilden kann. Bei beiden Methoden bleibt immer die Cardinalfrage, ob genügendes Material an Ureter und Blase vorliegt, bez. ob die Blase genügend beweglich ist, um eine Einpflanzung ohne eine für den Heilungsverlauf überaus missliche Dehnung und Zerrung zu ermöglichen.

Diese Vorbedingungen fehlen, wenn man den Ureter oberhalb der Linea innominata verletzt und ein grösseres Stück desselben mit einer Geschwulst (absichtlich oder unabsichtlich) entfernt hat. In derartigen Fällen, in welchen wegen des zu grossen Materialverlustes bei der Verletzung es unmöglich ist, den Ureter in die Blase einzupflanzen oder seine durchschnittenen Enden wieder zu nähern, wird man auf eine der nachstehenden Methoden zurückgreifen müssen. Diese, die nur einen Nothbehelf, auf den man gelegentlich in Ermangelung von Besserem angewiesen sein kann, bilden, sind: Gekreuzte Uretero-uretero-Anastomosis, Implantation in den Darm, Einnähung des: Ureterstumpfes in die Bauchdecken, Abbindung des'Ureters, Nephrektomie.

Die gekreuzte Uretero-uretero-Anastomosis (von Kelly und Wiesinger vorgeschlagen), die darin besteht, dass der für die Blasenimplantation und die Naht zu kurze Nierenstumpf des Ureters retroperitoneal über die Wirbelsäule gelegt und in den anderen Ureter eingenäht wird, ist bisher nur an Thieren ausgeführt. Dieses Verfahren bringt grosse Gefahren mit sich, da, falls die Einheilung 
nicht gelingt, die Continuität beider Ureteren zerstört werden würde; der sichere Tod wäre die Folge.

Die Harnleiterdarmeinpflanzung wurde vielfach an Thieren, zunächst mit unglücklichem Ausgang ausgeführt. Novaro war der Erste, der beim Hunde zeigte, dass es möglich ist, den Harnleiter in den Mastdarm einzupflanzen und dass die Nieren dabei gesund bleiben können. Auch Andere (Giordano, Virgnoni, Kalabin) hatten vereinzelte Erfolge; sie verloren aber eine sehr grosse Anzahl von Thieren.

Die ersten Operationen am Menschen verliefen gleichfalls ungünstig, bis Maydl (Wien. medic. Wochenschrift. 1894. No. 25 und 29) mit einer neuen Methode eine Heilung erzielte. Allerdings handelte es sich dabei nicht um die Wiederherstellung der Continuität durchtrennter Ureteren, vielmehr um einen Fall von Blasencarcinom, bei welcher die Blase ausgeschaltet werden sollte. Dabei nahm M. die Excision eines elliptischen Stückes der Blasenwand, welches die Ureterenmündungen enthielt, vor und nähte dieses excidirte Stück in den Mastdarm ein. Wenn auch weiterhin Krynsky und Virgnoni im Thierexperiment Verbesserungen des Maydl'schen Verfahrens ausarbeiteten, so kann doch keine einzige dieser Methoden irgendwelche Zukunft haben; denn wenn auch die Einheilung in den Darm erzielt sein sollte und es gelänge, dass keine zu starke Verengerung der Einpflanzungsstelle sich ausbildet, gegen die aufsteigende Infection hat man keinen Schutz. Fritsch (Veit's Handbuch der Gynäk. II. S. 146) schreibt über die Darmimplantation: "Die Idee, die Ureteren in den Darm einzunähen, ist als eine durchaus verfehlte zu betrachten. Dass sie technisch ausführbar ist, dürfte zweifellos sein, wie sie auch in der That ausgeführt ist. Aber die infectiöse aufsteigende Ureteritis, Pyelonephritis und Nephritis werden in jedem Fall über kurz oder lang eintreten müssen." Diese Argumentation ist so schlagend, dass man dieses Verfahren sicherlich nicht als legitime Methode empfehlen kann, wenn es auch Chaput einmal gelungen ist, bei einer Ureterenfistel nach vaginaler Hystereetomie den Ureter in den Darm und zwar in das Colon descendens mit Erfolg einzunähen.

Die Implantation des Ureters in die Bauchdecken ist eine Operation der Verlegenheit, durch welche ein Aufschub erreicht und der angerichtete Schaden mit geringem Zeitaufwand vorläufig in Ordnung gebracht wird. Als Fixationspunkt wählt man diejenige Stelle der Laparotomiewunde, in welche der Ureter mit der ge- 
ringsten Spannung hineingeleitet werden kann. Bei einer Bauchdeckenfistel machen sich aber ernstliche Zweifel bezüglich der weiteren normalen Function der betreffenden Niere geltend, da es gar zu leicht geschehen kann, dass bei einem geringen Versäumniss in der Pflege der Fistel pathogene Keime bis in die Niere vordringen, besonders wenn sich ein hartnäckiges Ekzem um die Ausflussöffnung etablirt hat, das einen vorzüglichen Nährboden für alle möglichen Mikroorganismen abgiebt. Nach Tauffer sah Bertini bei einer Bauchdeckenfistel die Niere 16 Monate lang gesund, musste aber sodann in Folge Nephritis die Nephrectomie vornehmen. Le Dentu und Pozzi beobachteten ebenfalls eine unter ähnlichen Umständen längere oder kürzere Zeit gesund verbliebene Niere (Ersterer durch 13 Tage, Letzterer sogar durch mehrere Monate). Da selbst bei ausbleibender Infection der Niere eine Ureterbauchdeckenfistel die Kranken durch den unaufhörlich ausfliessenden und irritirenden Urin den peinlichsten Belästigungen aussetzt, wird man gut thun, von diesem Verfahren nur im alleräussersten Notbfalle Gebrauch zu machen.

Die Abbindung des Ureters wurde schon öfter mit Erfolg zuerst an Thieren vorgenommen. Schon im Jahre 1874 wurde von Heidenhain ein interessanter Versuch gemacht, der beweist, dass nach plötzlichem Verschluss des Harnleiters die Secretion der betreffenden Niere sehr bald aufhört. H. unterband bei Thieren den Ureter und spritzte 24 Stunden später indigschwefelsaures Natron in's Blut. Nachdem eine Zeit lang blauer Urin entleert worden war, wurde das Thier getödtet. Während sich in der gesunden Niere, im Nierenbecken und Ureter grosse Massen von Farbstoff zeigten, befand sich an der operirten Seite in der Niere wenig, im Nierenbecken und Ureter garnichts von demselben.

Robinson (The results of partial and complete occlusion of the ureters, Ann. Surg. Phil., Vol XVIII, pag. 402) kommt bei seinen Versuchen über Verschluss und Ligirung des Ureters an Hunden zu dem Resultat, dass completer Verschluss zur Atrophie der Niere, incompleter dagegen zur Hydronephrose ${ }^{1}$ ) fübrt.

Lindemann (Ueber das Secretionsvermögen des Nierenparenchyms nach Harnleiterunterbindung, Centralblatt für allge-

1) Vergl. auch L. Landau, Ueber intermittirende Hydronephrose. Berl. klin. Wochenschr. 1888. No. 47. 
meine Path. und path. Anat. 1894, No. 11) studirte die Rückwirkung der Harnleiterunterbindung auf die Harnsecretion und fand drei Stadien von Nierenveränderungen: 1. venöse Hyperämie der Nieren, 2. Erweiterungen der Nierenkanälchen und 3. Nierenatrophie. Die Harnsecretion ist im ersten Stadium und noch mehr im zweiten vermindert, im dritten werden die Glomeruli für solche Stoffe durchgängig, die sonst nicht von ihnen ausgeschieden wurden, z. B. für Indigocarmin.

Bradford (Experim. atrophy of the kidney, Path. Soc. of London, Sitzung vom 7. 12. 1896, Lancet Vol Il, pag. 1533) erzielte bei Hunden durch Ligirung und Durehtrennung des Ureters eine Atrophie der Niere bis auf ein Viertel ihrer normalen Grösse. Die Atrophie beruhte auf Zusammengedrängtsein der Tubuli und Schrumpfung der Zellen.

Die Versuche von Büdinger (Archiv für klinische Chirurgie, Bd. 48, S. 639) und $\mathrm{Füth}$ zeigen, dass die Unterbindung des Ureters an sich technisch völlig sicher und ungefährlich ist

Auch nach Guyon (Retentions renales, Ann. des mal. des org. gen.-urin. 1891, Vol IX, No. 9) kann eine aseptische Nierenretention zu einer vollkommenen Nierenschrumpfung führen; el proponirte daher bei zufälligen Verletzungen des Ureters nach schwierigen Japarotomieen durch eine Unterbindung des Harnleiters eine aseptische Nierenretention zu erzeugen.

Es besteht freilich bei der Abbindung noch immer die Sorge, ob dic Ligatur auch dem Drucke des sich anstauenden Urins Stand hält. Füth hält dieses Bedenken, dass etwa Urin in die Bauchhöhle von dem unterbundenen Ureterstumpf austreten könnte, für unbegründet, weil er bei der Section eines Falles, bei welchem im Verlauf einer Laparotomie beide Ureteren unterbunden waren und die Frau an Urämie zu Grunde gegangen war, gesehen hatte; dass trotz colossaler Ausdehnung der Ureteren und der Nierenbecken kein Tropfen Urin in die Bauchhöhle gelangt war. Auch andere Fälle beweisen, dass die Unterbindung technisch völlig sicher ist. So der Fall von Purcell (s. o.), bei welchem nach 58 Stunden bei der secundären Laparotomie die Ligaturen, welche jederseits den Ureter fassten, gelöst wurden; auch hier kam kein Urin in die Bauchhöhle. Auch der Fall von Orthmann (Centralblatt für Gyn., Bd. XVII, S. 136) spricht für die Sicherheit der Ligatur. Orthmann beschreibt einen Fall von gabelförmig getheiltem linken Harnleiter, dessen eines Ende in normaler Weise in die Blase Archiv f. Gynäkologie. Bd. 65. H. 1. 
mündete, während das andere hinter der Harnröhre im Septum vesico-vaginale blind endigte und hier zur Bildung einer grossen Cyste Anlass gegeben hatte. Nach Eröffnung derselben, welche Orthmann vor der Abbindung des überzähligen Ureterastes vornahm, liess sich die Sonde ohne Schwierigkeit in der Richtung des Ureterverlaufes nach der linken Niere einführen. Nach sorgfältiger Unterbindung des freigelegten überzähligen Ureters wurde das untere Ende desselben nebst der cystischen Erweiterung abgetragen; das obere Ende zog sich gleich nach der Durchschneidung tief in das Becken hinein zurück. Heilung, keinerlei Beschwerden.

Bastianelli war der erste, der die Ligatur des Ureters am Menschen versuchte; in seinem und wahrscheinlich auch in $\mathrm{Phäno-}$ menow's Fall kam es zur Atrophie der Niere, in Füth's zwar zur Hydronephrose, die aber keinerlei Beschwerden machte. Auch in unserem Falle war der Verlaut ein sehr zufriedenstellender. Bis auf die dumpfeń Schmerzen im rechten Hypochondrium am 2. und 3. Tage post operationem traten weder subjective noch objective Störungen ein. Einen Tumor (Hydronephrose) konnten wir wäbrend der ganzen Zeit nicht palpiren, so dass es sehr wahrscheinlich ist, dass es zur Atrophie der Niere gekommen ist. Die cystoskopische Untersuchung am 20. October 1900 ergab: Links Ureterenwulst sichtbar und deutliches Spritzen des Ureters; rechts fehlt der Ureterwulst, die Ureteröffnung sehr klein, kaum sichtbar. Eine Urinentleerung an dieser Stelle nicht vorhanden. Uebrigens bis jetzt (Januar 1901) kein Recidiv.

Da in unserem Falle der Substanzverlust bei der Verletzung ein sebr grosser war, konnte man weder eine Blasenimplantation noch eine Vereinigung der Ureterenden vornehmen; selbst eine Implantation in die Bauchdecken wäre wegen der colossalen Dicke der letzteren einerseits und der Kürze des Ureters anderseits nicht ausführbar gewesen, und eine Implantation in den Darm wäre schon wegen der zu langen Dauer der Operation für die so ausgeblutete Patientin verhängsnissvoll geworden, ganz abgesehen von den Nachtheilen dieses Verfahrens an sich. Es blieb also nur die Wahl zwischen der Nephrectomie und der Abbindung des Ureters. Die Ligatur des Ureters wurde deshalb gewählt, weil man - abgesehen davon, dass man sich wegen Erschöpfung der Patientin (infolge des Eingriffs) nicht leicht zur Exstirpation der Niere entschlieșsen konnte - bei diesem Verfahren 
event. bei nicht hinreichender Function der anderen Niere eine Nierenbeckenfistel an der Niere, deren Ureter durchschnitten ist, hätte anlegen können. Dadurch war gleichsam ein Sicherheitsventil gegen eine event. drohende Urämie gegeben. Auf die Nachtheile dieser Methode musste man freilich gefasst sein, vor Allem darauf, in längerer oder kürzerer Zeit eine Nephrektomie zu machen. Die Indication zur letzteren wäre gegeben, sobald es zur Pyonephrose gekommen wäre, oder sonst eine Sackbildung in der Niere bedrohliche Erscheinungen gemacht hätte.

Wie bereits oben erwähnt, war es in keinem der vier Fälle von Ligatur des Ureters nöthig, secundär eine Exstirpation der Niere vorzunehmen. Aber selbst, wenn in derartigen Fällen eine nach der. Unterbindung entstandene Pyonephrose eine Exstirpation erheischen sollte, ist die Prognose besser als bei der primären Nephrectomie. Nach Tauffer (Dieses Archiv, Bd. 46, S. 552) ist die zweizeitige Nierenexstirpation der einzeitigen vorzuziehen, wenn es auch sehr unangenehm ist, die Kranke nach Ueberstehen der durch die Operation bedingten Gefahr neuerdings einer nicht ungefährlichen erneuten Operation auszusetzen. Taulfer erscheint die secundäre Nephrectomie deshalb weniger gefährlich als die primäre, weil die zweite Niere nicht auf einmal, nicht mit einem Schlag, sondern allmälig die Function der anderen übernimmt; die Theorie Favre's wie auch seine eigene klinische Erfahrung führten ihn zu dieser Annahme. Favre (Virchow's Archiv 1892, Bd. CXXVII, S. 33) bewies nämlich an Thierexperimenten, dass die Ursache der nach Nephrektomieen auftretenden, relativ grossen Sterblichkeit in den meisten Fällen in einer Nephritis parenchymatosa acutissima zu finden ist, die aus einer specifischen Ptomainämie zu erklären ist. Die plötzlich belastete zurückbleibende Niere ist nicht im Stande, die nöthige vicariirende Function sofort ganz zu übernehmen," so dass die Bakterien und deren Producte sich anhäufen und einen heftigen Reizzustand in der Niere verursachen. Auf diese Weise gingen von Favre's nephrektomirten Kaninchen $66 \mathrm{pCt}$. zu Grunde. Unterband Favre dagegen nur den Harnleiter - eine Operation, bei welcher 12 pCt. der Versuchsthiere zu Grunde gingen - und exstirpirte erst 14. Tage darauf die entsprechende Niere, so blieben alle Versuchsthiere am Iueben. Aus diesen experimentellen Resultaten schliesst Favre, dass durch vorhergehende Unterbindung des einen Ureters die andere Niere zu genügender compensirender Thätigkeit heranwächst, so dass die nachher voll- 
180 Weinreb, Ein Beitrag zur Therapie der Ureterenverletzungen etc.

zogene Nierenexstirpation vom Organismus sehr leicht ertragen werden kann, indem die zweite Niere ohne Störung die Aufgabe der gesammten Urinausscheidung übernimmt. Er schlägt daher vor, die Nephrektomie in zwei Zeiten zu machen und glaubt dadurch die Mortalität der Nephrektomie herabmindern zu können. - Auf Grund dieser Beobachtungen erscheint es bei der Wahl zwischen Nephrectomie und Ligatur des Ureters gerechtfertigt, den Ureter zu ligiren, wenn es auch später zur Nephrectomie kommen sollte.

Aber selbst in solchen Fällen, in denen man eine Bauchdeckenfistel anlegen kann, ist zu erwägen, ob nicht die Unterbindung des Ureters vorzuziehen ist, da man der Patientin bei diesem Verfahren die Nephrectomie ersparen kann, während eine Bauchdeckenfistel - wie bereits erwähnt - fast stets früher oder später die Nephrectomie nöthig macht.

Anders bei bereits bestehender Infection des Ureters und des Nierenbeckens. In derartigen Fällen ist die Anlegung einer Bauchdeckenfistel indicirt, die Unterbindung kein rationelles Verfahren, da es unchirurgisch ist, eine inficirte Höhle zu verschliessen und dadurch den freien Abfluss zu versperren. Der Vorzug der Ureterunterbindung, dass man nämlich der Patientin eine Nierenexstirpation event. ersparen kann, kommt in derartigen Fällen deswegen nicht in Betracht, weil eine Pyonephrose im Körper nicht verbleiben kann.

Empfehlenswerth ist es, nach dem Vorschlag Stoeckel's (Ureterfisteln und Ureterverletzungen, Leipzig 1900) den abgebundenen Ureter in die Haut einzunähen. Bei diesem Verfahren ist es einerseits ganz ausgeschlossen, dass Urin in die Bauchhöhle fliesst, andererseits kann eine drohende Urämie sehr leicht behoben werden, indem man die Ligatur löst. 